\title{
Analysis of seized stanozolol formulations in South Brazil by liquid chromatography coupled to quadrupole time-of-flight-mass spectrometry
}

\author{
Everton R. Campos ${ }^{\mathrm{a}, 1}$, Sarah Eller ${ }^{\mathrm{a}, 1}$, Letícia Birk ${ }^{\mathrm{a}}$, Masurquede A. Coimbra ${ }^{\mathrm{a}}$, Sandra M.D. Macedo ${ }^{\mathrm{a}, \mathrm{b}}$, Mauricio \\ Yonamine $^{\mathrm{c}}$, Josias O. Merib ${ }^{\mathrm{a}}$, Tiago F. Oliveira ${ }^{\mathrm{a}, *}$
}

\begin{abstract}
${ }^{a}$ Pharmacosciences Department, Federal University of Health Sciences of Porto Alegre, Porto Alegre, Brazil; ${ }^{b}$ Instituto Nacional de Ciência e Tecnologia Forense (INCT Forense), Porto Alegre, Brazil; ' School of Pharmaceutical Sciences, University of São Paulo, São Paulo - SP, 05508-000, Brazil
\end{abstract}

*Corresponding author: oliveira@ufcspa.edu.br

${ }^{1}$ These authors contributed equally to this work.

\begin{abstract}
Anabolic-androgenic steroids (AAS) are synthetic derivatives of testosterone which are used medically for several diseases. However, misuse is commonly observed by athletes to promote enhancement of strength, bodyweight and performance. AAS are frequently obtained by internet black market from clandestine drug manufacturing laboratories, without any quality standards, being potentially dangerous for users. The purpose of this work was the development and application of a fast and simple procedure for the quantitation of stanozolol by liquid chromatography coupled to quadrupole time-offlight-mass spectrometry (LC-QTOF-MS) in tablets packs seized in Rio Grande do Sul state, Brazil. The samples of stanozolol were separated considering its pharmaceutical forms. An internal standard was added to the aliquots of the samples, dissolved in methanol and $5 \mu \mathrm{L}$ were injected into the analytical system. The newly developed method has been validated for lower limit of quantitation (LLOQ), linearity, accuracy, precision and selectivity. The lower limit of quantification (LLOQ) was $0.1 \mu \mathrm{g} / \mathrm{mL}$. The developed method was successfully applied to 31 samples seized by the Secretaria da Receita Federal do Brasil (a Brazilian federal revenue service agency). According to the results, $90.3 \%$ of the suspected medicines $(n=31)$ were adulterated, and $65 \%$ exhibited higher concentrations of stanozolol than those indicated in the label. This work successfully established a new method for quantification of stanozolol using LC-QTOFMS. This method aims at contributing to the identification and quantification of this anabolic androgenic steroid frequently seized by federal inspection agencies.
\end{abstract}

Keywords: Anabolic-androgenic steroids, stanozolol, liquid chromatography, mass spectrometry. https://doi.org/10.22456/2527-2616.108853

\section{Introduction}

Anabolic-androgenic steroids (AAS) are synthetic derivatives of testosterone and they are used medically for the treatment of hypogonadism, metabolic disorders, burns, anemia, bone marrow failure, chronic renal failure and acquired immunodeficiency syndrome $(1,2)$. However, misuse is commonly observed by athletes to promote strength increase, bodyweight and performance gain $(1,3)$. Adverse events were associated with a variety of different cardiovascular diseases (4-6), liver disorders including peliosis hepatis, hepatocellular hyperplasia and carcinomas (7) and a spectrum of behavioral effects correlated with hyperandrogenism (8).

Legitimate pharmaceutical grade AAS are illegally acquired in pharmacies. In addition, black marketed AAS have frequently been obtained by internet from clandestine drug manufacturing laboratories, without any quality standards. In both cases, these products can be potentially harmful for users promoting intoxication $(9,10)$

In Brazil, studies indicate that erectile dysfunction medicines, AAS and anorexigenic products are the most commonly counterfeit and contraband drugs (11). Brazilian Federal Police reported that, from 2006 to 2011, one third of the 3,537 AAS analyzed products were counterfeit products (12). Additionally, Neves et al. conducted the analysis of 345 AAS positive medicines/dietary supplements samples and observed that $53 \%$ were substandard, spurious, falsely labelled, falsified or counterfeit (SSFFC) medical products (13). The measurements conducted by Ribeiro et al. indicated that $20 \%$ of seized AAS samples analyzed showed adulterants not described in the labels. Among those, stanozolol samples presented the most unusual results since none of the capsules had the analyte presence, showing adulteration with other AAS compounds and sildenafil citrate (14).

Stanozolol (STZ) is an alkylated C17 $\alpha$ steroid commonly detected SSFFC medical product in Brazilian scenario (9). It is a heterocyclic steroid derived from dihydrotestosterone, containing chemical alterations in order to increase anabolic activity and decrease androgenic pathways, resulting in and anabolic/androgenic ratio of 320/30 $(15,16)$. Because of its anabolic characteristics, STZ can stimulate protein and erythropoietin synthesis. Therefore, it promotes body fat loss while preserving the lean mass to achieve the physical shape desired (17). Despite all the beneficial outcomes, STZ presents side effects such as most of the AAS, even with their low androgenicity. Voice changes, alopecia, hair growth, clitoris increase, and many others 
have been reported. STZ particularly exhibits a high hepatotoxic activity, since the presence of the alkyl in C17-alpha reduces liver metabolization.

Stanozolol analytical measurements can be conducted by several techniques. This compound can be determined in several biological matrices such as blood, plasma, serum, urine, hair, nails and oral fluid for doping control purposes (18). Also, it can be evaluated in pharmaceutical formulations in order to determine its authenticity. In this scenario, chromatographic approaches are the most established methodologies (14). Gas chromatography coupled to mass spectrometry (MS) is widely used for screening, while liquid chromatography coupled to MS or tandem mass spectrometry (MS/MS) ensures the required analysis precision, specificity and sensibility with minimal sample pretreatment $(18,19)$. Moreover, spectroscopic methods such as proton nuclear magnetic resonance (1H NMR), Raman and Fourier transform infrared spectroscopy (FT-IR) can be applied to the identification and quantitation of stanozolol $(14,20)$. In addition, the use of high-resolution mass spectrometry (HRMS) methods is suggested due to the ability to improve mass accuracy and enhance the selectivity of the target compounds and/or allow for the analysis of untargeted compounds.

The aim of the present study was to evaluate the quality of formulations containing the anabolic steroid stanozolol, seized by the Secretaria da Receita Federal do Brasil (a Brazilian federal revenue service agency). For this purpose, a liquid chromatography coupled to quadrupole time-of-flight-mass spectrometry (LCQTOF-MS) method was developed and validated, being applied to the analysis of 31 stanozolol formulations.

\section{Materials and Methods}

\section{Chemical and reagents}

Methanolic solutions of stanozolol and methyltestosterone (internal standard; IS) at a concentration of $1.0 \mathrm{mg} / \mathrm{mL}$ were obtained from Cerilliant Analytical Reference Standards (Round Rock, TX, USA). HPLC-grade methanol, acetonitrile and formic acid were purchased from Merck (Darmstadt, Germany). Water was purified using a Milli-Q system (Millipore, Billerica, MA, USA). All reference standards were $>99 \%$ purity.

\section{Preparation of standard solutions}

Stock solutions of stanozolol and IS were prepared at concentrations of $200 \mu \mathrm{g} / \mathrm{mL}$ and $10 \mu \mathrm{g} / \mathrm{mL}$ respectively, using methanol. These solutions were stored under refrigeration $\left(2-8 \quad{ }^{\circ} \mathrm{C}\right)$. Working solutions with concentrations between 0.1 and $20 \mu \mathrm{g} / \mathrm{mL}$ were also prepared in methanol by diluting stock solutions.

\section{Chromatographic conditions and data acquisition}

A micrOTOF-Q III (Bruker Daltonics, Bremen, Germany) coupled to a Shimadzu Prominence liquid chromatography system (Shimadzu, Kyoto, Japan) was used for the analyses. Mass data were obtained with the following optimized conditions: capillary voltage, 3000 $\mathrm{V}$; nebulizer gas, 3 bar; drying gas, $5 \mathrm{~L} / \mathrm{min}$; drying temperature, $150{ }^{\circ} \mathrm{C}$; quadrupole ion energy, $4 \mathrm{eV}$; mass range, $\mathrm{m} / \mathrm{z} 50$ - 350 acquired with $1 \mathrm{~Hz}$. The system was calibrated in positive mode using a solution of sodium formate $(10 \mathrm{mM})$ and the protonated molecular ions $\mathrm{m} / \mathrm{z}$ 329.2584 (stanozolol) and $\mathrm{m} / \mathrm{z} \quad 303.2325$ (methyltestosterone) were detected with a mass error of $<5 \mathrm{ppm}$. The following chromatographic condition was used: a $75 \times 2.0 \mathrm{~mm}$ i.d., $2.2 \mu \mathrm{m}$, Shim-pack XRODS II (Shimadzu, Kyoto, Japan) column eluted with a gradient of $0.1 \%$ formic acid in water (A) and acetonitrile (B) at a $400 \mu \mathrm{L} / \mathrm{min}$ flow rate and $45^{\circ} \mathrm{C}$ as follows: $0-2.5$ min, $40-100 \%$ of $\mathrm{B} ; 2.5-3.5 \mathrm{~min}, 100 \%$ of $\mathrm{B} ; 3.5-3.6$ $\min , 100-40 \%$ of $\mathrm{B} ; 3.6-5 \mathrm{~min}, 40 \%$ of $\mathrm{B}$.

\section{Sample preparation and analyses}

Analyses were performed using stanozolol formulations samples seized by the Secretaria da Receita Federal do Brasil. Sample classification was executed in order to discriminate tablet from capsules, resulting in 31 packs ( $\mathrm{n}=29 \times 100$ tablets; $\mathrm{n}=2 \times 50$ capsules). The tablets packs were manufactured by a Paraguayan laboratory which has no health license in Brazil. The capsule packs exhibited no manufacturing nor expiration dates, only a handwritten identification of the active ingredient, concentration and number of capsules. The means of the weights of each batch were determined thought the individual measurements of 20 tablets and capsules (with and without the content). For the individual weight evaluation, one tablet/capsule of each pack was weighted and compared to the means obtained. This procedure was carried out according to the recommendations of the $32^{\text {nd }}$ edition of the American Pharmacopeia (USP 43) (21). Afterwards, a $1 \mathrm{mg}$ were weighed of the tablets/capsules on an analytical balance (Shimadzu, AUW220D; $0.1 \mathrm{mg} / 0.01 \mathrm{mg}$ ) was transferred to falcon tubes and diluted with $4 \mathrm{~mL}$ of methanol, followed by homogenization in vortex for $30 \mathrm{~s}$ and centrifugation for $10 \mathrm{~min}$ at $3500 \mathrm{rpm}$. For quantitative analysis IS working solution $(100 \mu \mathrm{g} / \mathrm{mL})$ was added to all samples and an aliquot of $5 \mu \mathrm{L}$ was directly injected into the analytical system.

\section{Optimization of the Q-TOF parameters}

In this step, a multivariate approach strategy based on a central composite design was applied for the optimization of three parameters that are relevant in order to obtain the maximum analytical response of the target compound: capillary voltage $(1320 \mathrm{~V} ; 2200 \mathrm{~V} ; 3500 \mathrm{~V} ; 4800 \mathrm{~V} ; 5680$ $\mathrm{V})$, drying temperature $\left(150{ }^{\circ} \mathrm{C} ; 190^{\circ} \mathrm{C} ; 250^{\circ} \mathrm{C} ; 310^{\circ} \mathrm{C}\right.$; $\left.350^{\circ} \mathrm{C}\right)$ and collision energy $(1 \mathrm{eV} ; 6 \mathrm{eV} ; 13 \mathrm{eV} ; 20 \mathrm{eV}$; $25 \mathrm{eV})$. A total of 17 experiments were evaluated using as response the absolute area provided by the analyte in all examined conditions. The software Statistica (Statsoft, 
EUA) was used to evaluate the experimental design through a response surface methodology.

\section{Validation}

The method was validated according to international guidelines and recommendations for validation of analytical methods, by establishing parameters such as the lower limit of quantitation (LLOQ), linearity, accuracy, precision and selectivity (22-25). The study of linearity was performed by injecting working standard solutions of the analytes at six levels: $0.1,1,5,10,15$ and $20 \mu \mathrm{g} / \mathrm{mL}$, in six replicates for each concentration. Precision and accuracy were performed through the analysis of three different levels $(0.2,8$ and $16 \mu \mathrm{g} / \mathrm{mL})$. Six replicates on each of three consecutive days were acquired and the precision data (within and between-day) were calculated using one-way analysis of variance (ANOVA). Selectivity was evaluated in the lowest level of precision, adding compounds that may be present in the sample (ephedrine, caffeine and sibutramine).

\section{Results and Discussion}

\section{Optimization of the Q-TOF parameters}

The response surfaces obtained with the variable's capillary voltage, temperature and collision energy are shown in Figure 1. According to the results, the optimized results of capillary voltage, temperature and collision energy are respectively $3000 \mathrm{~V}, 150{ }^{\circ} \mathrm{C}$ and $4 \mathrm{eV}$. These values represent the highest analytical signal intensity for stanozolol, and they were used for subsequent validation and analyses. The use of multivariate approaches to determine the optimal conditions in experimental procedures is a valid and effective strategy. Traditional univariate approaches can be comprised of several empirical experiments, which can make the analysis timeconsuming and more expensive.
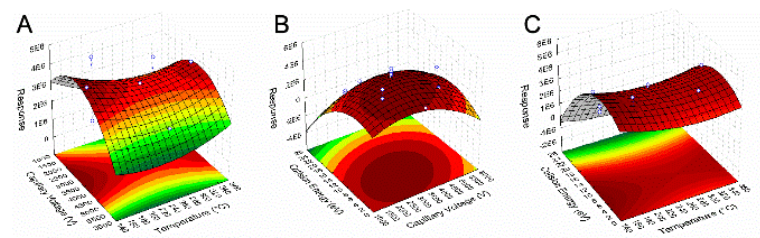

Figure 1. Response surfaces (peak area) for the evaluation of the Q-TOF parameters: a) Capillary Voltage (V) x Temperature $\left({ }^{\circ} \mathrm{C}\right)$; b) Collision Energy $(\mathrm{eV}) \times$ Capillary Voltage $(\mathrm{V})$; c) Collision Energy $(\mathrm{eV}) \mathrm{x}$ Temperature $\left({ }^{\circ} \mathrm{C}\right)$.

\section{Validation}

The LLOQ value was determined by empirical method, which consists of analyzing a series of samples concentrations of the analyte, and the LLOQ obtained was $0.1 \mu \mathrm{g} / \mathrm{mL}$ (Figure 2). The calibration curve was linear over the specified range $(0.1-20 \mu \mathrm{g} / \mathrm{mL})$. The linear regression equation and the respective coefficient of determination were $y=0.0018 x+0.1653, r^{2}=0.9975$. The analyte has presented heteroscedastic calibration curve, which may be explained by the wide range of concentration values considered in this parameter. Therefore, linear least squares regression methods have resulted in large errors in the calculation of the concentrations, especially at lower values. By applying weighted least squares linear regression, the resulting weighting factor was of $1 / y$ and the linear regression equation applied was $y=1.141319 x-0.09435\left(r^{2}=\right.$ $0.9919)$.

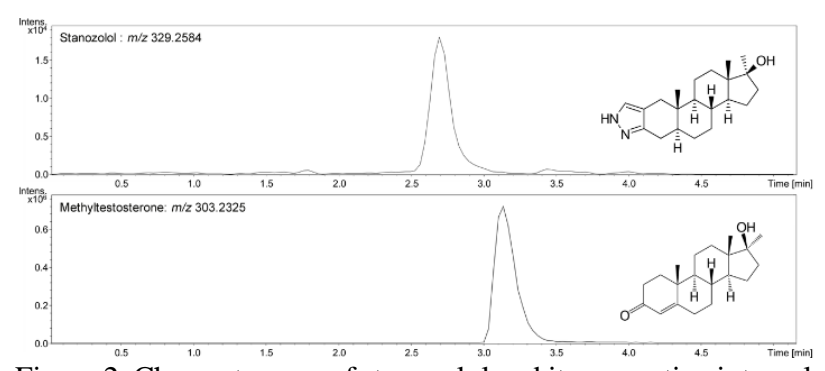

Figure 2. Chromatogram of stanozolol and its respective internal standard (methyltestosterone) at LLOQ value $(0.1 \mu \mathrm{g} / \mathrm{mL})$.

The method selectivity was performed under the specified test conditions and no significant level of interfering at the retention time of the analyte was observed, thus confirming the selectivity of the method. The precision was assessed by ANOVA and the results obtained of intra-day precision presented a variation of $11.4-17.8 \%$. For inter-day precision the results were achieved in the range of 11.8 to $16.7 \%$. Results on precision and accuracy of the validated method are shown in Table 1 .

Table 1. Precision and accuracy data of the developed method for stanozolol formulations at three quality control (QC) levels: QC Low $(0.2 \mu \mathrm{g} / \mathrm{mL})$, QC Medium $(8 \mu \mathrm{g} / \mathrm{mL})$, QC High $(16$

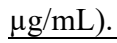

\begin{tabular}{cll} 
Parameters & $\begin{array}{c}\text { Quality Controls } \\
\text { (QC) }\end{array}$ & Results \\
\hline \multirow{2}{*}{ Accuracy (\%) } & QC Low & 92.67 \\
& QC Medium & 95.24 \\
& QC High & 92.10 \\
\hline \multirow{2}{*}{ Intra-day precision (\% } & QC Low & 17.79 \\
CV) & QC Medium & 12.05 \\
& QC High & 11.04 \\
\hline \multirow{2}{*}{ Inter-day precision (\% } & QC Low & 16.68 \\
CV) & QC Medium & 13.88 \\
& QC High & 13.69 \\
\hline
\end{tabular}

CV: Coefficient of variation

\section{Samples analysis}

The means of the weight of tablets and capsules, so as the individual weights of each sample and the variation are summarized in Table 2. It was noted that 5 samples (4 tablets and 1 capsule) exceeded the variation limit of $\pm 5 \%$ for tablets and $\pm 7.5 \%$ for capsules established by the regulatory agencies. These variations can directly influence on the content of stanozolol in the product compromising its quality. 
Table 2. Quality control data for mean weight calculated by batch, individual weights and variation. NM: Not mentioned

\begin{tabular}{|c|c|c|c|c|c|}
\hline Batch & $\begin{array}{l}\text { Sample } \\
\text { number }\end{array}$ & $\begin{array}{l}\text { Batch } \\
\text { weight } \\
\text { (mg) }\end{array}$ & $\begin{array}{c}\text { Mean } \\
\text { weight } \\
\text { (mg) }\end{array}$ & $\begin{array}{c}\text { Weight } \\
\text { variation } \\
(\mathrm{mg})\end{array}$ & $\begin{array}{c}\text { Weight } \\
\text { variation } \\
(\%)\end{array}$ \\
\hline \multicolumn{6}{|c|}{ Tablets } \\
\hline 16116 & 14 & 252.50 & \multirow{9}{*}{249.55} & 2.95 & $1.18 \%$ \\
\hline 16116 & 15 & 266.81 & & 17.26 & $6.92 \%$ \\
\hline 16116 & 18 & 240.09 & & -9.46 & $-3.79 \%$ \\
\hline 16116 & 19 & 257.92 & & 8.37 & $3.35 \%$ \\
\hline 16116 & 21 & 232.87 & & -16.68 & $-6.68 \%$ \\
\hline 16116 & 23 & 263.59 & & 14.04 & $5.63 \%$ \\
\hline 16116 & 24 & 255.95 & & 6.40 & $2.56 \%$ \\
\hline 16116 & 26 & 250.59 & & 1.04 & $0.42 \%$ \\
\hline 16116 & 27 & 257.62 & & 8.07 & $3.23 \%$ \\
\hline 16098 & 1 & 257.32 & \multirow{2}{*}{250.25} & 7.07 & $2.83 \%$ \\
\hline 16098 & 22 & 244.54 & & -5.71 & $-2.28 \%$ \\
\hline 16097 & 4 & 250.48 & 255.63 & -5.15 & $-2.01 \%$ \\
\hline 16074 & 12 & 259.04 & \multirow{2}{*}{249.92} & 9.12 & $3.65 \%$ \\
\hline 16074 & 13 & 245.62 & & -4.30 & $-1.72 \%$ \\
\hline 16063 & 2 & 242.85 & \multirow{4}{*}{254.45} & -11.60 & $-4.56 \%$ \\
\hline 16063 & 7 & 234.15 & & -20.30 & $-7.98 \%$ \\
\hline 16063 & 9 & 261.60 & & 7.15 & $2.81 \%$ \\
\hline 16063 & 10 & 265.55 & & 11.10 & $4.36 \%$ \\
\hline 16046 & 25 & 258.84 & 258.23 & 0.61 & $0.24 \%$ \\
\hline 16045 & 3 & 258.01 & \multirow{4}{*}{255.58} & 2.43 & $0.95 \%$ \\
\hline 16045 & 11 & 257.00 & & 1.42 & $0.56 \%$ \\
\hline 16045 & 16 & 257.78 & & 2.20 & $0.86 \%$ \\
\hline 16045 & 28 & 255.12 & & -0.46 & $-0.18 \%$ \\
\hline 16043 & 6 & 266.10 & 256.87 & 9.23 & $3.59 \%$ \\
\hline 16041 & 5 & 244.31 & \multirow{2}{*}{250.94} & -6.63 & $-2.64 \%$ \\
\hline 16041 & 20 & 254.53 & & 3.59 & $1.43 \%$ \\
\hline 16017 & 8 & 248.65 & 250.01 & -1.36 & $-0.54 \%$ \\
\hline 16013 & 17 & 258.70 & \multirow{2}{*}{256.26} & 2.44 & $0.95 \%$ \\
\hline 16013 & 29 & 258.41 & & 2.15 & $0.84 \%$ \\
\hline \multicolumn{6}{|c|}{ Capsules } \\
\hline NM & 30 & 378.78 & 393.53 & -14.75 & $-3.75 \%$ \\
\hline NM & 31 & 378.87 & 413.18 & -34.31 & $-8.30 \%$ \\
\hline
\end{tabular}

After the validation of the LC-QTOF-MS method, the concentration of the active ingredient of stanozolol in each tablet and capsule was calculated. The reference parameter of variation of the active ingredient concentration was the monograph of stanozolol described in the $32^{\text {nd }}$ edition of the American Pharmacopeia (21). This document defines that stanozolol tablets should not contain less than $90.0 \%$ or more than $110.0 \%$ of the labeled amount. Quantitative results for each sample analyzed are shown in Table 3. Variation of the content measurements are presented in the control graph (Figure $3)$.
Table 3. Concentration results for the analyses of stanozolol formulations.

\begin{tabular}{|c|c|c|c|c|}
\hline Batch & $\begin{array}{l}\text { Sample } \\
\text { number }\end{array}$ & $\begin{array}{l}\text { Concentration } \\
\text { indicated on } \\
\text { the label (mg) }\end{array}$ & $\begin{array}{l}\text { Individual } \\
\text { weight } \\
\text { (mg) }\end{array}$ & $\begin{array}{c}\text { Concentration } \\
\text { measurements } \\
\text { by LC-HRMS } \\
\text { (mg) }\end{array}$ \\
\hline \multicolumn{5}{|c|}{ Tablets } \\
\hline 16116 & 14 & 10 & 252.50 & 11.72 \\
\hline 16116 & 15 & 10 & 266.81 & 13.85 \\
\hline 16116 & 18 & 10 & 240.09 & 10.56 \\
\hline 16116 & 19 & 10 & 257.92 & 16.95 \\
\hline 16116 & 21 & 10 & 232.87 & 14.79 \\
\hline 16116 & 23 & 10 & 263.59 & 11.64 \\
\hline 16116 & 24 & 10 & 255.95 & 12.38 \\
\hline 16116 & 26 & 10 & 250.59 & 11.71 \\
\hline 16116 & 27 & 10 & 257.62 & 11.01 \\
\hline 16098 & 1 & 10 & 257.32 & 10.65 \\
\hline 16098 & 22 & 10 & 244.54 & 13.04 \\
\hline 16097 & 4 & 10 & 250.48 & 8.06 \\
\hline 16074 & 12 & 10 & 259.04 & 8.14 \\
\hline 16074 & 13 & 10 & 245.62 & 14.34 \\
\hline 16063 & 2 & 10 & 242.85 & 22.49 \\
\hline 16063 & 7 & 10 & 234.15 & 7.70 \\
\hline 16063 & 9 & 10 & 261.60 & 8.12 \\
\hline 16063 & 10 & 10 & 265.55 & 13.99 \\
\hline 16046 & 25 & 10 & 258.84 & 13.28 \\
\hline 16045 & 3 & 10 & 258.01 & 12.98 \\
\hline 16045 & 11 & 10 & 257.00 & 13.56 \\
\hline 16045 & 16 & 10 & 257.78 & 7.54 \\
\hline 16045 & 28 & 10 & 255.12 & 13.57 \\
\hline 16043 & 6 & 10 & 266.10 & 4.27 \\
\hline 16041 & 5 & 10 & 244.31 & 10.33 \\
\hline 16041 & 20 & 10 & 254.53 & 18.16 \\
\hline 16017 & 8 & 10 & 248.65 & 8.33 \\
\hline 16013 & 17 & 10 & 258.70 & 13.48 \\
\hline 16013 & 29 & 10 & 258.41 & 13.25 \\
\hline \multicolumn{5}{|c|}{ Capsules } \\
\hline NM & 30 & 20 & 378.78 & 30.06 \\
\hline NM & 31 & 250 & 378.87 & 23.29 \\
\hline
\end{tabular}

According to Figure 3, only three samples are within the limit of variation, with results similar than the labeled concentration of the seized material. Also, inaccuracy of the informed concentration of stanozolol has been found, since some of the samples exhibited double or half the concentration indicated on the package. Moreover, it was noticed a significant heterogeneity of the content in 28 seized samples of stanozolol, and only 3 samples are within the concentration range regulated by the American Pharmacopeia (USP 43) (21)

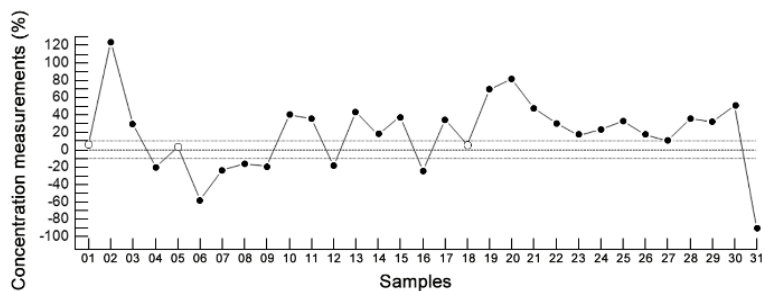

Figure 3. Control graph of the variation of stanozolol according to the limits established by the $32^{\text {nd }}$ edition of the American Pharmacopeia. The dotted lines indicated the limits in which the concentration should be ranging. 
According to the results, $90.3 \%$ of the samples appear to be adulterated. Of this percentage, $65 \%$ exhibited higher concentrations than those indicated on the package. In this study, the presence of other non-related substances has not been observed. These results exhibit correlation with other studies conducted in Brazil. Berneira et al. observed $25 \%$ of counterfeit and $62.5 \%$ of adulterated formulations of several AAS including stanozolol (26). Ribeiro et al. observed three main types of adulteration of AAS: absence of the active ingredient, presence of other compounds and concentrations below those indicated. Stanozolol injectable solutions presented contents below the half of the declared on the label. Moreover, the capsules have shown no presence of stanozolol and the existence of other compounds (14). Neves et al. has shown that $53 \%$ of the AAS were counterfeit including stanozolol samples that did not present the active ingredient and exhibited lower or higher amount than the indicated (13). The main difference between this study and other data already published consisted of the adulterated samples evaluated exhibited much higher concentrations than those declared on the label. This discrepancy can be correlated with the potential increase of the AAS side effects already described in the literature. In addition, this fact can contribute in intoxication and overdosage processes in athletes, bodybuilders and gym attenders, considering the multiple cycles and drugs used by these public.

\section{Conclusions}

This work successfully established a new LC-QTOF-MS method for stanozolol analysis. This method aimed at contributing to the identification and quantitation of the anabolic/androgenic steroid stanozolol frequently seized by federal inspection agencies. Quality control analyses were performed in 31 samples seized by the Secretariat of the Federal Revenue of Brazil by the evaluation of the means and individual weights of tablets and capsules and the content measurement. It was shown that $16.13 \%$ of samples reproved the quality control for weight and $90.3 \%$ were adulterated. Considering only the adulterated samples, $65 \%$ have shown a higher concentration than the one indicated on the label. This study presents concerning data about the quality of AAS acquired in black markets (e.g. academies and/or internet) which can be responsible for several side effects and intoxications.

\section{Acknowledgments}

The authors would like to acknowledge the financial support from Coordination of Improvement of Personal Higher Education - Brazil [CAPES - Finance Code 001].

\section{Conflict of interest}

The authors declare no conflicts of interest.

\section{References}

1.He G, Wu Y, Lu J. Doping control analysis of 13 steroids and structural-like analytes in human urine using Quadrupole-Orbitrap LC-MS/MS with parallel reaction monitoring (PRM) mode. Steroids. 2018; 131:1-6.

2.Cunha TS, Cunha NS, Moura MJCS, Marcondes FK. Esteróides anabólicos androgênicos e sua relação com a prática desportiva. Rev Bras Cienc Farm. 2004; 40:165179.

3.Anti-doping testing figures. World Anti-Doping Agency (WADA). https://www.wadaama.org/en/resources/laboratories/anti-doping-testingfigures (accessed July 02, 2019).

4. P. Vanberg, D. Atar, Androgenic anabolic steroid abuse and the cardiovascular system, Handb. Exp. Pharmacol. 195 (2010) 411-457.

5.F.R. De Souza, A.R.K. Sales, M.R. Dos Santos, R.A. Porello, G.W.P. Fonseca, A.L.C. Sayegh, et al., Retrograde and oscillatory shear rate in young anabolic androgenic steroid users, Scand J Med Sci Sports. 29 (2018) 422-429.

6.De Souza FR, Dos Santos MR, Porello RA, et al. Diminished cholesterol efflux mediated by HDL and coronary artery disease in young male anabolic androgenic steroid users. Atherosclerosis. 2019; 283:100-105.

7.Hartgens F, Kuipers H. Effects of Androgenic-Anabolic Steroids in Athletes. Sport. Med. 2004; 34:513-554.

8.Cunningham RL, Lumia AR, McGinnis MY. Androgenic anabolic steroid exposure during adolescence: ramifications for brain development and behavior. Horm. Behav. 2013; 64:350-356.

9.Fact Sheet \& Substandard, spurious, falsely labelled, falsified and counterfeit (SSFFC) medical products. World Health Organization (WHO) http://www.who.int/medicines/regulation/ssffc/en/ (accessed July 02, 2019).

10.Fink J, Schoenfeld BJ, Hackney AC, et al. Anabolicandrogenic steroids: procurement and administration practices of doping athletes. Physician Sports Med. 2019; 47:10-14.

11.Hurtado RL, Lasmar MC. Counterfeit and contraband drugs in Brazil: overview and prospects for preventing. Cad. de Saude Publica. 2014; 30:891-895.

12.Neves DBDJ, Marcheti RG, Caldas ED. Incidence of anabolic steroid counterfeiting in Brazil. Forensic Sci. Int. 2013; 228:81-83. 
13.Neves DBDJ, Caldas ED. GC-MS quantitative analysis of blackmarket pharmaceutical products containing anabolic androgenic steroids seized by the Brazilian Federal Police. Forensic Sci. Int. 2017; 275:272-281.

14.Ribeiro MVM, Boralle N, Felippe LG, Pezza HR, Pezza L. ${ }^{1} \mathrm{H}-\mathrm{NMR}$ determination of adulteration of anabolic steroids in seized drugs. Steroids. 2018; 138:47-56.

15.Llewellyn's W. Anabolics: The bestselling anabolic reference, 11th edition. Molecular Nutrition (2011) $832 \mathrm{p}$.

16.Metabocard for stanozolol. Human Metabolome Database (HMDB). http://www.hmdb.ca/metabolites/HMDB0003116 (accessed July 02, 2019).

17.Tsitsimpikou C, Tsarouhas K, Spandidos DA, Tsatsakis MA. Detection of stanozolol in the urine of athletes at a pg level: The possibility of passive exposure. Biomed. Rep. 2016; 5:665-666.

18.Gosetti F, Mazzucco E, Gennaro MC, Marengo E. Ultra high-performance liquid chromatography tandem mass spectrometry determination and profiling of prohibited steroids in human biological matrices. A review. J Chromatogr B Analyt Technol Biomed Life Sci. 2013; 15:22-36.

19.Pellegrini M, Rotolo MC, Di Giovannadrea R, Pacifici R, Pichini S. A simple toxicological analysis of anabolic steroid preparations from the black market. Annales de Toxicologie Analytique. 2012; 24:67-72.

20.Lemma T, Souza FB, Souto CAT, Martin AA. An FTRaman, FT-IR, and Quantum Chemical Investigation of Stanozolol and Oxandrolone. Biosensors. 2018; 8:214.

21.USP 43 NF 58: United States pharmacopeia [and] national formulary. United States Pharmacopeian Convention.

22.Guidance for the Validation of Analytical Methodology and Calibration of Equipment used for Testing of Illicit Drugs in Seized Materials and Biological Specimens. United Nations Office of Drugs and Crime (UNODC). https://www.unodc.org/documents/scientific/validatio n_E.pdf (accessed July 02, 2019).

23.Peters FT, Maurer HH. Bioanalytical method validation and its implications for forensic and clinical toxicology - A review. Accredit. Qual. Assur. 2002; $7: 441-449$.
24.Peters FT, Drummer OH, Musshoff F. Validation of new methods. Forensic Sci. Int. 2007; 17:216-224.

25.Scientific Working Group for Forensic Toxicology (SWGTOX), Standard Practices for Method Validation in Forensic Toxicology. J. Anal. Toxicol. 2013; $37: 452-474$.

26.Berneira LM, Freitas SC, Silva CC, Machado AM, Pereira CMP, Santos MAZ. Application of differential scanning calorimetry in the analysis of apprehended formulations of anabolic androgenic steroids. Forensic Sci. Int. 2019; 296:15-21. 